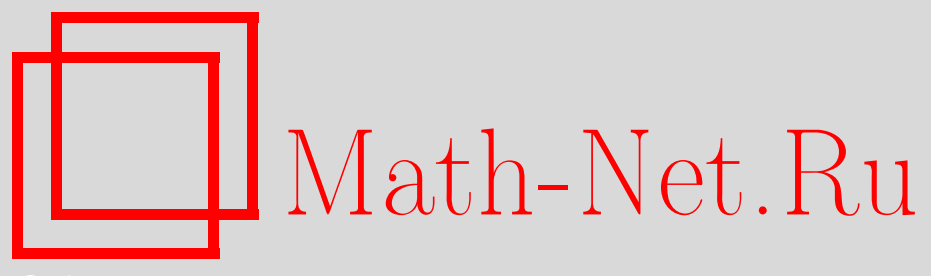

В. В. Беняш-Кривец, В. П. Платонов, Непрерывные дроби и $S$-единицы в гиперэллиптических полях, $\mathrm{y} M H$, 2008, том 63, выпуск 2, 159-160

DOI: https://doi.org/10.4213/rm9184

Использование Общероссийского математического портала Math-Net.Ru подразумевает, что вы прочитали и согласны с пользовательским соглашением http://www . mathnet.ru/rus/agreement

Параметры загрузки:

IP : 35.173 .219 .12

26 апреля 2023 г., 12:50:49

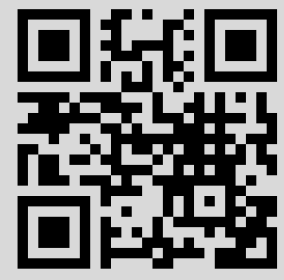




\section{Непрерывные дроби и $S$-единицы в гиперэллиптических полях}

\section{В. В. Беняш-Кривец, В. П. Платонов}

Настоящая заметка преследует двойную цель: изложить некоторые результаты о непрерывных дробях в функциональных полях и показать, как непрерывные дроби могут быть использованы для нахождения фундаментальных $S$-единиц в гиперэллиптических полях.

Пусть $k$ - произвольное поле, $k(x)$ - поле рациональных функций от одной переменной над $k$. Для многочлена $v=x-a$ через $|\cdot|=|\cdot|_{v}$ будем обозначать нормирование, соответствующее $v$. Пополнение поля $k(x)$ относительно нормирования $|\cdot|_{v}$ можно отождествить с полем формальных степенных рядов $k((v))$. Продолжение нормирования $|\cdot|$ на $k((v))$ по-прежнему будем обозначать через $|\cdot|$.

Непрерывные дроби в функциональных полях в случае нормирования $|\cdot|_{\infty}$ были впервые введены Э. Артином [1]. Мы рассматриваем случай нормирования $|\cdot|_{v}$. Для элемента $\beta=\sum_{i=-s}^{\infty} d_{i} v^{i} \in k((v))$ положим $[\beta]=\sum_{i=-s}^{0} d_{i} v^{i} \in k\left[v^{-1}\right]$. Пусть $a_{0}=[\beta]$. Если $\beta-a_{0} \neq 0$, то положим $\beta_{1}=1 /\left(\beta-a_{0}\right) \in k((v)), a_{1}=\left[\beta_{1}\right]$. Далее по индукции определяем элементы $a_{i}, \beta_{i}$ : если $\beta_{i-1}-a_{i-1} \neq 0$, то $\beta_{i}=1 /\left(\beta_{i-1}-a_{i-1}\right), a_{i}=\left[\beta_{i}\right]$. Этот процесс оборвется тогда и только тогда, когда $\beta \in k(v)$. Будем использовать стандартную сокращенную запись для непрерывной дроби $\beta=\left[a_{0} ; a_{1}, a_{2}, \ldots\right]$.

Определим по индукции элементы $p_{i}, q_{i} \in k\left[v^{-1}\right]$. Положим $p_{-2}=0, p_{-1}=1$, $q_{-2}=1, q_{-1}=0$ и если $n \geqslant 0$, то $p_{n}=a_{n} p_{n-1}+p_{n-2}, q_{n}=a_{n} q_{n-1}+q_{n-2}$. Тогда $p_{n}, q_{n} \in k\left[v^{-1}\right]$ и $p_{n} / q_{n}=\left[a_{0} ; a_{1}, \ldots, a_{n}\right]$ при $n \geqslant 0$. Для $n \geqslant-1$ справедливы соотношения

$$
q_{n} p_{n-1}-p_{n} q_{n-1}=(-1)^{n}, \quad q_{n} \beta-p_{n}=\frac{(-1)^{n}}{q_{n} \beta_{n+1}+q_{n-1}}, \quad \beta=\frac{p_{n} \beta_{n+1}+p_{n-1}}{q_{n} \beta_{n+1}+q_{n-1}} .
$$

Дробь $p_{n} / q_{n}$ называют $n$-й подходящей дробью к $\beta$. Нетрудно показать, что

$$
\lim _{n \rightarrow \infty} \frac{p_{n}}{q_{n}}=\beta .
$$

По построению, $\left|a_{n}\right|=\left|\beta_{n}\right|<0$. Из (1) по индукции легко получить соотношения

$$
\left|q_{n}\right|=\sum_{j=1}^{n}\left|a_{j}\right|, \quad\left|q_{n} \beta-p_{n}\right|=-\left|q_{n+1}\right|>-\left|q_{n}\right| .
$$

Введем понятие наилучшего приближения. Дробь $p / q$, где $p, q \in k\left[v^{-1}\right], q \neq 0$, является наилучшим приближением к $\beta$, если для любой другой дроби $a / b$, где $a, b \in k\left[v^{-1}\right], b \neq 0$, такой, что $a / b \neq p / q$ в $k(v)$ и $|b| \geqslant|q|$, выполнено неравенство $|\beta-p / q|>|\beta-a / b|$. Справедливо

Предложение 1. Несократимая дробъ $p / q$, где $p, q \in k\left[v^{-1}\right], q \neq 0$, является наилучшим приближением $\kappa \beta$ тогда и только тогда, когда $|\beta-p / q|>-2|q|$ (или, что эквивалентно, $|q \beta-p|>-|q|)$.

Предложение 1 и соотношения (2) немедленно влекут, что $n$-я подходящая дробь $p_{n} / q_{n}$ к $\beta$ является наилучшим приближением к $\beta$. Следующая теорема показывает, что справедливо и обратное утверждение.

Теорема 1. Если а/b является наилучшим приближением $\kappa \beta$, то найдутся такая подходящая дробъ $p_{n} / q_{n} \kappa \beta$ и такая константа $c \in k^{*}$, что $a=c p_{n}, b=c q_{n}$.

Стандартным образом можно показать, что если непрерывная дробь $\left[a_{0} ; a_{1}, a_{2}, \ldots\right]$ для $\beta$ является периодической, то $\beta$ - квадратичная иррациональность. В случае бесконечного поля $k$ обратное утверждение верно не всегда [2]. Всюду ниже мы будем 
предполагать, что $k=\mathbb{F}_{q}$ - поле из $q$ элементов и характеристика $k$ не равна 2 . Справедливо следующее предложение.

ПредЛОЖениЕ 2. Если $\beta \in k((v))$ - квадратичная иррачиональность, то непрерывная дробъ для $\beta$ периодична.

Далее мы покажем, как непрерывные дроби могут быть использованы для нахождения фундаментальных $S$-единиц в гиперэллиптических полях. Пусть $d(x)=$ $b_{0} x^{2 n+1}+b_{1} x^{2 n}+\cdots+b_{2 n+1} \in k[x]$, где $b_{0} \neq 0,-$ свободный от квадратов многочлен, $K=k(x)(\sqrt{d})$. Предположим, что наше нормирование $|\cdot|=|\cdot|_{v}$ имеет два продолжения $|\cdot|_{1}$ и $|\cdot|_{2}$ на $K$. Нормирование $|\cdot|_{\infty}$ имеет единственное продолжение на $K$. Пусть $S=\left\{|\cdot|_{\infty},|\cdot|_{1}\right\}, \mathcal{O}_{S}$ - кольцо $S$-целых элементов в $K, U_{S}=\mathcal{O}_{S}^{*}-$ группа $S$-единиц поля $K$. Известно, что группа $U_{S}$ является прямым произведением группы $k^{*}$ и свободной абелевой группы $G$ ранга 1 . Образующая группы $G$ называется фундаментальной $S$-единицей.

В [3] найден эффективный алгоритм для вычисления фундаментальной $S$-единицы. В классическом случае квадратичного расширения $L=\mathbb{Q}(\sqrt{d})$ поля $\mathbb{Q}$ фундаментальную единицу поля $L$ можно найти, используя разложение $\sqrt{d}$ либо $(\sqrt{d}-1) / 2$ в непрерывную дробь. Наша цель - показать, что и в случае гиперэллиптического поля $K$ фундаментальную $S$-единицу можно найти, используя метод непрерывных дробей. В [3] доказано, что для вычисления фундаментальной $S$-единицы нужно найти минимальное натуральное $m$ такое, что норменное уравнение

$$
f^{2}-g^{2} d=a v^{m}
$$

где $a \in k^{*}$, имеет решение в многочленах $f, g \in k[v], g \neq 0$. Тогда либо $f+g \sqrt{d}$, либо $f-g \sqrt{d}$ является фундаментальной $S$-единицей. Следующая теорема дает алгоритм для нахождения фундаментальной $S$-единицы с помощью непрерывных дробей.

Теорема 2. Пусть $m$ - такое минимальное натуралъное число, что норменное уравнение (3) имеет решение в многочленах $f, g \in k[v], g \neq 0$.

1. Если $m$ нечетно, то $f / g=p_{n} / q_{n}$ для некоторой подходящей дроби $p_{n} / q_{n} \kappa \sqrt{d}$.

2. Если $m=2 t$ четно, то найдется делитель $h$ многочлена $d$, обладающий следующими свойствами: i) $1 \leqslant \operatorname{deg} h \leqslant(\operatorname{deg} d-1) / 2$; іi) уравнение

$$
h f_{1}^{2}-\frac{d}{h} g_{1}^{2}=b v^{t}
$$

где $b \in k^{*}$, имеет решение в многочленах $f_{1}, g_{1} \in k[v]$, при этом $f_{1} / g_{1}=p_{n} / q_{n}$ для некоторой подходящей дроби $p_{n} / q_{n} \kappa \sqrt{d} / h$. Наоборот, если $f_{1}, g_{1} \in k[x]-$ решение уравнения (4), то многочлены $f$ u $g$, определяемые по формулам $f=h f_{1}^{2}+(d / h) g_{1}^{2}$, $g=2 f_{1} g_{1}$, являются решением норменного уравнения (3).

\section{Список литературы}

[1] E. Artin, Math. Z., 19:1 (1924), 153-206. [2] W. W. Adams, M. J. Razar, Proc. London Math. Soc. (3), 41:3 (1980), 481-498. [3] В. В. Беняш-Кривец, В. П. Платонов, Докл. РАН, 417:4 (2007), 446-450; англ. пер.: V. V. Benyash-Krivets, V.P. Platonov, Dokl. Math., 76:3 (2007), 886-890.

\section{В. В. Беняш-Кривец (V. V. Benyash-Krivets)}

Белорусский государственный университет

E-mail: benyash@bsu.by

\section{В. П. Платонов (V.P. Platonov)}

Научно-исследовательский институт

системных исследований РАН

E-mail: platonov@niisi.ras.ru
Представлено Д. В. Аносовым Принято редколлегией 12.02.2008 